\title{
Preoperative Geriatric Nutritional Risk Index is an independent prognostic factor for postoperative survival after Gallbladder carcinoma radical surgery
}

\section{Die Zhang}

The second affiliated hospital and Yuying children's hospital of wenzhou medical university Jing Xu ( $\nabla$ xujing_6@126.com)

The second affiliated hospital and Yuying children's hospital of wenzhou medical university https://orcid.org/0000-0002-7543-8774

\section{Research article}

Keywords: Geriatric Nutritional Risk Index, gallbladder cancer, survival, nutritional, prognosis

Posted Date: April 12th, 2020

DOI: https://doi.org/10.21203/rs.3.rs-21722/v1

License: (c) (1) This work is licensed under a Creative Commons Attribution 4.0 International License.

Read Full License 


\section{Abstract}

Background Surgical results of gallbladder cancer (CBG) are currently inadequate. The Geriatric Nutritional Risk Index (GNRI) can effectively assess nutritional status. The present study attempted to delve into investigating whether preoperative GNRI can act as a predicting factor for CBG. Methods 202 consecutive GBC patients treated from 2010 to 2017 were taken retrospectively. By conducting univariate and multivariate Cox regression analyses on overall survival (OS) and recurrence-free survival (RFS), their preoperative GNRI was studied. Results Of the 202 patients, 103 (51.0\%) had GNRI<100. The patients with low GNRI before surgery had the median OS of 26 months, less than that of patients having received high GNRI before surgery (39 months, $\mathrm{P}<0.001$ ). GNRI displayed associations with less survival times in univariate $(\mathrm{HR}, 3.656 ; 95 \% \mathrm{Cl}, 2.308-5.790 ; \mathrm{P}<0.001)$. As indicated from the results of multivariate analysis, patients exhibiting low GNRI showed a lower OS (HR, 2.386; 95\%Cl, 1.170-4.868; $\mathrm{P}=0.017)$ and RFS (HR, 7.610; 95\% Cl, 3.181-18.207; $P<0.001)$ than those exhibiting high GNRI. Conclusions GNRI is an independent indicator of poor prognosis in GBC patients after GBC radical surgery.

\section{Introduction}

Gallbladder cancer (GBC) has been the commonest malignant biliary tract tumor, taking up $80 \%-95 \%$ of biliary cancer patients [1]. CBG refers to the fifth commonest malignancy of the gastrointestinal tract, taking up 1\% of all cancers in our nation[2]. Because of insufficient effective diagnostic markers, single treatment choices, late diagnosis, and atypical symptoms, the prognosis of GBC remains ineffective[3]. GBC prognosis has been primarily determined by the pathologic detecting process of cancer tissue and identification of the pathologic type, classification, and stage of cancer for patients' prognosis. As indicated from the results of epidemiologic studies, patients carrying this disease achieved $30 \%$ and $5 \%$ 3 - and 5-years survival rates, respectively[4]. Thus, generally considering the ineffective prognosis for $\mathrm{GBC}$, other factors affecting the survival of this type of patients should be identified.

The association between survival of patients carrying organ malignancies (e.g., gallbladder cancer) and the nutritional status is a research hotspot. Some research demonstrated that preoperative nutritional status showed associations with postoperative complications, as well as long-term results of malignant tumors patients[5-7]. The serum albumin concentration acts as an index of protein reserves. In recent years, a growing number of researches proved that low serum albumin are critical prognosis predictors in many cancers. In contrast, body mass index (BMI) acts as a reasonable adiposity measure. Elevated BMI refers to a risk factor for death by cancer, stroke, and ischemic heart disease[8].

The Geriatric nutritional risk index (GNRI) refers to an indicator of malnutrition, capable of acting as an uncomplicated and important factor for predicting the results achieved from only serum albumin and the rate between ideal and practical body weights. Such index has been recently analyzed in a range of studies; its prognostic value was demonstrated for patients on diffuse large B cell lymphoma, esophageal cancer, cardiovascular diseases and chronic liver failure[9-12]. Note that, whether the GNRI is feasible to cure organ malignancies (e.g., GBC) is still unclear. 
Accordingly, this study hypothesizes that the GNRI acts as a prognosis factor of GBC patients after they undergo surgery. The present study attempted to delve into whether preoperative GNRI can be a prognosis factor for survival in GBC patients in a retrospective manner.

\section{Material And Methods}

A retrospective analysis is carried out in this study following the Helsinki Declaration and approved by the Ethics Committee of the Second Affiliated Hospital of Wenzhou Medical University. The written informed consent of all subjects was obtained. Eligible patients were involved in this study, including (1) patients with histological diagnosis of GBC; (2) GBC patients with no other coexisting malignancies; (3) patients not receiving other treating processes before recruitment; (4) patients with available follow-up data and overall clinical data: and (5) patients aged $>18$ years. The exclusion criteria included (1) patients exhibiting acute infection or chronic active inflammatory disease; (2) patients with anemia, collagen diseases and others regarding the hematological mechanism; (3) patients having taken anticoagulant treatment or albumin transfusions before receiving the treatment; (5) patients exhibiting perioperative surgery-associated mortality. Clinical data within 30 days before surgery for 202 of the patients were included. At the Second Affiliated Hospital, Wenzhou Medical University, all patients received radical operation for CBG for 2010-2017. This study made phone calls to the patients once a year to record their recent physical situation. The follow-up data included the data of surgery to the death date or last contacting, or the end of November 2019. The dates from the surgery to those of the last follow-up or death referred to overall survival (OS). Recurrence free survival (RFS) was computed according to the date of surgery till the initial relapse or death for certain reasons, or the date of last following-up.

\section{Clinical information}

The medical information were harvested from the patients in a retrospective manner, covering TNM staging (The United States Joint Committee on cancer [7th edition], staging of gallbladder cancer), pathologic data (e.g., tumor differentiation, tumor size, tumor lymph node metastasis), serum carcinoembryonic antigen (CEA), serum CA19-9, neutrophil-to-lymphocyte ratio (NLR), serum albumin, hemoglobin, BMI, age, and gender. Based on electrochemiluminescence immunoassays (Cobas; Roche Diagnostics, Germany) performed at the Clinical Laboratory Department, Second Affiliated Hospital of Wenzhou Medical University, China, serum CA19-9 and CEA levels were identified here. The normal reference values in line with a previous study are as follows: CEA $\leq 5 \mathrm{ug} / \mathrm{L}, \mathrm{CA} 19-9 \leq 37 \mathrm{U} / \mathrm{mL}, \mathrm{NLR}<2.6[13]$. The radical resection is defined that the primary tumor is removed together with the affected tissue and the metastatic lymph nodes. (For the T1 a GBC, by carrying out a laparotomy or laparoscopic surgery, a simple cholecystectomy was performed. For the T1b GBC, an extended cholecystectomy was employed. For patients with GBC in stage T2 or above, an extended cholecystectomy is frequently performed. In the extended cholecystectomy, IVb/V liver resection or the gallbladder bed wedge resection could be conducted following the intraoperative condition. The scope of lymph node dissection should cover the posterior superior pancreaticoduodenal lymph node, the hepatic artery and portal vein lymph nodes, the 
lymph nodes around the hepatoduodenal ligament the common bile duct lymph node, as well as the cystic duct lymph node. According to the tumor pathology, there was no tumor tissue at the margin.

\section{GNRI calculation}

We acquired GNRI from individually obtained serum albumin levels, ideal body weight, actual body weight in $\mathrm{kg}$, and height in $\mathrm{cm}$, as shown below [14]:

GNRI $=(1.489 *$ albumin $(\mathrm{g} / \mathrm{L}))+(41.7 *($ weight/WL0) $)$,

where WLO refers to the ideal weight, obtained by:

male: WL0=H-100-((H-150)/4)

female: $\mathrm{H}-100-((\mathrm{H}-150) / 2.5)$

where $\mathrm{H}$ denotes the height.

With the mentioned values of GNRI, two grades of risk in relation to nutrition were defined: high risk $(<100)$ and low risk $(\geq 100)$

\section{Statistical method}

For categorical variables, by the chi-suqared test or Fisher's exact test, noticeable diversification were assessed. For continuous data, by independent-sample t-test or the Mann-Whitney test, the mean diversification was compared. To find the associations between OS and parameters, univariate and multivariate Cox proportional hazard models were recruited here. OS was obtained as the time from the data of surgery to the date of the last follow-up or death from any cause. With the use of the KaplanMeier approach, we studied survival curves. By conducting the log-rank test, the relevant comparison was drawn. A p-value $<0.05$ was identified noticeable from the statistical perspective. Statistical analyses were conducted with the use of SPSS version 22 (SPSS, Lnc., Chicago, IL, USA).

\section{Results}

\section{Baseline characteristics}

202 patients suffering GBC were recruited in general, the mean GNRI was $100.08 \pm 10.69,68.3 \%$ were female, and the mean age reached $68.54 \pm 11.02$ years. Besides, $51 \%$ patients exhibited low GNRI with $\mathrm{GBC}$ according to diagnosing processes. The fundamental characteristics of survivor and non-survivor patients are listed in Table 1. In comparison to survivor patients, non-survivor patients were older, with lower GNRI, albumin, hemoglobin and BMI. High CA19-9 $(P<0.001)$ and high CEA $(P=0.006)$ between the survivor and non-survivor patients were considerably divergent. Regarding tumor characteristics, advanced infiltration depth T $(P<0.001)$, advanced TNM stage $(P<0.001)$, tumor differentiation $(P<0.001)$ and lymph node metastasis $(P=0.046)$ considerably impacted the death of $G B C$. Besides, the GNRI helped 
to split participants into groups with high GNRI ( $n=99)$ and low GNRI $(n=103)$ (Table 2). In contrast to high GNRI patients, the low GNRI patients had larger age, carrying lower albumin, hemoglobin, BMI. Higher NLR $(P<0.001)$, CA19-9 $(P=0.028)$, incidence of gallstones $(P=0.048)$ and poorer tumor differentiation $(P=0.046)$ exerted considerable effect at low GNRI levels.

\section{Kaplan-Meier survival analysis}

Among the 202 cases, 94 died and 108 survived. For the OS and RFS $(P<0.001)$ (Fig. 1), the prognosis was considerably worse in patients exhibiting poor GNRI levels than in patients exhibiting high GNRI levels. Patients in the high GNRI level group exhibited a medium OS time of 39 months ( $95 \% \mathrm{Cl} 29.78-$ 48.22), and patients exhibiting the low GNRI showed a mean OS of 26 months ( $95 \% \mathrm{Cl} 20.46-31.55)$. The recurrence rate during following-up here reached $52.0 \%$ (105 cases), and the middle illness -free survival in this series reached 24 months. The middle RFS was noticeably smaller in the low GNRI group than in the high GNRI group (12months and 35 months, separately; $\mathrm{P}<0.01)$.

\section{Univariate and multivariate analysis}

The OS-related variables after GBC radical surgery complying with univariate and multivariate Cox proportional hazard models are listed in Table 3 . In the univariate analysis, age, serum CA19-9>37, CEA $>5$, hemoglobin level, invasive depth III+IV, poor differentiation, lymph node metastasis and GNRI<100 displayed associations with low OS. According to the results of multivariate analysis, age (HR, 1.030, 95\% Cl, 1.001-1.061; $\mathrm{P}=0.045)$, hemoglobin ( $\mathrm{HR}, 1.025,95 \% \mathrm{Cl}, 1.001-1.049 ; \mathrm{P}=0.043)$, poor differentiation $(\mathrm{HR}, 2.073,95 \% \mathrm{Cl}, 1.079-4.391 ; \mathrm{P}=0.047)$, TNM III+IV (HR, 8.182, 95\%Cl, 1.727-38.776; $\mathrm{P}=0.008)$ and low GNRI (HR, 2.386, 95\% Cl, 1.170-4.868; $\mathrm{P}=0.017)$ adversely affected $\mathrm{OS}$. Table 4 lists a multiple-variate and univariate Cox proportional hazard regression mode for RFS. By multivariate analysis, this study identified two adverse prognosis elements, affecting RFS, namely, TNM stage (HR, 3.236, 95\% $\mathrm{Cl}, 1.051$ $11.117 ; \mathrm{P}=0.049)$ and $\mathrm{GNRI}(\mathrm{HR}, 7.610,95 \% \mathrm{Cl}, 3.181-18.207 ; \mathrm{P}<0.001)$.

\section{Prognostic value of GNRI}

To draw the comparison of the predictability of the mortality and recurrence rate among GBC patients, ROC curves for BMI, albumin, GNRI were plotted (Fig 2). GNRI exhibits the highest area underneath the ROC curves, being statistically obvious (AUC $=0.739$ for mortality, $95 \% \mathrm{Cl}, 0.670-0.808 ; \mathrm{P}<0.001$, AUC $=0.716$ for recurrence rate, $95 \% \mathrm{Cl}, 0.644-0.787 ; \mathrm{P}<0.001)$. The optimal cutoffs of GNRI for predicted mortality and recurrence rate reached 100.40 and 100.15 separately.

\section{Discussion}

GBC refers to a rare disease, the commonest incursive tumor in the biliary mechanism[1, 15].

Nevertheless, GBC progress rapidly, developing rare early symptom[16]. Accordingly, detection in advance and surgical treating process in advance are upheld [15], whereas GBC prognostic process is still not 
effective. Accordingly, novel biomarkers for the prognosis of GBC are required. Low GNRI was identified a less effective predicting factor of OS and RFS in GBC patients after they underwent GBC radical surgery.

Serum albumin level and BMI are critical variables capable of reflecting the survival risk in malignancies patients. Serum albumin refers to a highly related malnutrition indicator, effectively indexing cachexia or malnutrition in cancer patients, since this factor is partly suggested by hypoalbuminemia. Nutrition can be employed as a critical factor to determine immune response[17]; For this reason, hypoalbuminemia may show associations with damaged immune response. In comparison, a systematic review revealed that high serum albumin concentration displayed associations with higher cancer patients' surviving condition [18].

Though a number of works demonstrated an association between obesity and risk of GBC incidence [19], these are also studies that weight of body loss refers to an ineffective prognosis factor of CBG. According to Jinwoo Kang, survival was considerably better in patients exhibiting large BMI in comparison to those exhibiting poor BMI. Furthermore, body weight loss was expected under negative cell regulating mechanisms for cancer or in patients of aggressive cancer. Nevertheless, low BMI or body weight loss refers to a negative prognosis factor for GBC patients[20].

According to Kawai et al., nutrition critically determines immune responses, and malnutrition most frequently leads to immunodeficiency[21]. Furthermore, malnutrition displayed associations with cellmediated immunity damage for the host defenses resisting cancer. Malnutrition is a state of proteinenergy or other nutritional imbalance, as well as a feature of cachexia. Systemic inflammation in the course of tumor progression will result in cachexia in cancer patients. In cancer patients with cachexia, elevated circulating concentrations of a range of inflammatory cytokines were identified[22]. Given the mentioned results, malnutrition is likely to confirm poor outcome even in GBC patients resected in a surgical manner.

The GNRI as relying on body weight loss and serum albumin concentration reveals the nutritional status. Accordingly, the GNRI can assess both of the mentioned variables in the meantime. In addition, GNRI can be simply obtained according to laboratory data achieved routinely and can assess the nutritional status. In fact, in Fig. 2, GNRI exhibited the largest area under ROC curve in comparison to those of BMI and albumin, which is noticeable from the perspective of statistics. Nevertheless, few studies have verified the clinics-based use of GNRI for organ malignancies, expect esophageal cancer[11].

In this study, approximate $51 \%$ of patients had GNRI less than 100 , though each case appeared to be in sufficient health, according to these patients' preoperative functional tests results and appearance, for undergoing surgical resection. As revealed by the mentioned result, preoperative GNRI can be adopted to effectively to identifying patients undernourished among all patients with appropriate organ functions and allowed to receive surgery.

In the present study, compared with patients exhibiting large GNRI before surgery, patients exhibiting poor GNRI exhibited larger NLR $(P<0.001)$, CA19-9 $(P=0.028)$, incidence of gallstones $(P=0.048)$ and poorer 
tumor differentiation. Accordingly, patients exhibiting poor GNRI were likely to show tumor development. For this reason, such variable is likely to be an effective index of high-grade tumor malignancy. Nevertheless, this study cannot draw the conclusion that low GNRI is likely to lead to or be attributed to tumor malignancy according to this work.

Furthermore, by multivariate analysis, poor differentiation, low hemoglobin, high age, low GNRI, lymph node metastases and TNM III + IV were identified as separate prognostic factors in GBC patients. Considering our knowledge, it was first reported in this study that a preoperative low GNRI is a predictive and prognostic factor of GBC, since no existing analyses reported that GNRI is feasible to treat other cancers. Moreover, it was reported that low GNRI patients had considerably shorter OS than high GNRI patients. Accordingly, GBC and low GNRI patients are at high-risk of death even after undergoing therapeutic processes. For this reason, after this type of patients have undergone surgeries, more careful follow-up should be conducted.

Bo et al. reported that the GNRI estimated survival in old esophageal cancer patients received radiotherapy[11]. Given the conclusion drawn by these researchers, the GNRI was a single prognosis factor in elderly esophageal cancer patients underwent radiotherapy. The mentioned data supported the findings of this study as well.

The present study has several limits. Firstly, a retrospective study was carried out in an individual institution, and bias existed, in particular bias of selection. Nevertheless, such bias was restricted by including consecutive patients in a set period. Secondly, in a single cohort, the GNRI's prognosis implication was tested, as well as its cutoff value. Though the study tends to be overfitting, no other cohort can be presented, or our cohort cannot be split to a training and verification cohort. The results of this study are supposed to be verified in larger single cohorts. Irrespective of the mentioned limits, the first data are presented, revealing the GNRI as a vital prognosis factor in GBC patients. Since the GNRI includes available parameters, the GNRI applies to standard clinical settings.

\section{Conclusions}

To sum up, the preoperative GNRI refers to a new predicting factor and prognosis factor; it can be used to find out which GBC patients should undergo multimodality therapies. A subsequent study should be conducted to assess the survival enhancement of multimodality therapies for GBC patients having low GNRI.

\section{Abbreviation}

GNRI, geriatric nutritional risk index; GBC, gallbladder cancer; OS, overall survival; CEA, carcinoembryonic antigen; NLR, neutrophil-to-lymphocyte ratio

\section{Declarations}




\section{Funding}

This work was supported by the grants of Project of Zhejiang Provincial Department of Health(2016KYB194), General Research Project of Zhejiang Provincial Education

Department(Y201534290), Wenzhou Science and Technology Bureau(Y20120163). The funders played no role in the design of this study, the collection, analysis and interpretation of data or preparation of the manuscript.

\section{Competing interests}

The authors declare no potential conflicts of interest.

\section{Acknowledgments}

The authors thank the staff at the Second Affiliated Hospital and Yuying Children's Hospital of Wenzhou Medical University, and all the patients who participated in the study.

\section{Acknowledgments}

The authors thank the staff at the Second Affiliated Hospital and Yuying Children's Hospital of Wenzhou Medical University, and all the patients who participated in the study.

\section{Consent for publication}

Not applicable

\section{Author Contributions Statement}

JX - study concept and design; preparation, review and approval of manuscript. DZ - data collection and interpretation; preparation, review and approval of manuscript.

\section{Ethics approval and consent to participate}

This study has obtained the approval from the Ethics Committee of the Second Affiliated Hospital of Wenzhou Medical University (No. LCKY2017-21) and has obtained the written informed consent of all subjects following the Declaration of Helsinki.

\section{Availability of data and materials}

The data that support the findings of this study are available from Institutional Review Board of the second affiliated hospital and Yuying Children's Hospital of Wenzhou Medical University but restrictions apply to the availability of these data, which were used under license for the current study, and so are not publicly available. Data are however available from the authors upon reasonable request and with permission of Institutional Review Board of the Second Affiliated Hospital and Yuying Children's Hospital of Wenzhou Medical University. 


\section{References}

1. Lazcano-Ponce EC, Miquel JF, Munoz N, Herrero R, Ferrecio C, Wistuba II, et al. Epidemiology and molecular pathology of gallbladder cancer. Cancer J Clin. 2001;51:349-64.

2. Legan M. Cyclooxygenase-2, p53 and glucose transporter-1 as predictors of malignancy in the development of gallbladder carcinomas. Bosnian journal of basic medical sciences. 2010;10:192-6.

3. Xu WY, Zhang HH, Yang XB, Bai Y, Lin JZ, Long JY, et al. Prognostic significance of combined preoperative fibrinogen and CA199 in gallbladder cancer patients. World journal of gastroenterology. 2018;24:1451-63.

4. Hu MT, Wang JH, Yu Y, Liu C, Li B, Cheng QB, et al. Tumor suppressor LKB1 inhibits the progression of gallbladder carcinoma and predicts the prognosis of patients with this malignancy. Int $\mathrm{J}$ Oncol. 2018;53:1215-26.

5. Lien YC, Hsieh CC, Wu YC, Hsu HS, Hsu WH, Wang LS, et al. Preoperative serum albumin level is a prognostic indicator for adenocarcinoma of the gastric cardia. Journal of gastrointestinal surgery: official journal of the Society for Surgery of the Alimentary Tract. 2004;8:1041-8.

6. Schwegler I, von Holzen A, Gutzwiller JP, Schlumpf R, Muhlebach S, Stanga Z. Nutritional risk is a clinical predictor of postoperative mortality and morbidity in surgery for colorectal cancer. $\mathrm{Br} \mathrm{J}$ Surg. 2010;97:92-7.

7. Alifano M, Mansuet-Lupo A, Lococo F, Roche N, Bobbio A, Canny E, et al. Systemic inflammation, nutritional status and tumor immune microenvironment determine outcome of resected non-small cell lung cancer. PloS one. 2014;9:e106914.

8. Nakagawa T, Toyazaki T, Chiba N, Ueda Y, Gotoh M. Prognostic value of body mass index and change in body weight in postoperative outcomes of lung cancer surgery. Interact Cardiovasc Thorac Surg. 2016;23:560-6.

9. Nakagomi A, Kohashi K, Morisawa T, Kosugi M, Endoh I, Kusama Y, et al. Nutritional Status is Associated with Inflammation and Predicts a Poor Outcome in Patients with Chronic Heart Failure. J Atheroscler Thromb. 2016;23:713-27.

10. Taniguchi E, Kawaguchi T, Otsuka M, Uchida Y, Nagamatsu A, Itou M, et al. Nutritional assessments for ordinary medical care in patients with chronic liver disease. Hepatology research: the official journal of the Japan Society of Hepatology. 2013;43:192-9.

11. Bo Y, Wang K, Liu Y, You J, Cui H, Zhu Y, et al. The Geriatric Nutritional Risk Index Predicts Survival in Elderly Esophageal Squamous Cell Carcinoma Patients with Radiotherapy. PloS one. 2016;11:e0155903.

12. Kanemasa Y, Shimoyama T, Sasaki Y, Hishima T, Omuro Y. Geriatric nutritional risk index as a prognostic factor in patients with diffuse large B cell lymphoma. Annals of hematology. 2018;97:999-1007.

13. Pang Q, Zhang LQ, Wang RT, Bi JB, Zhang JY, Qu K, et al. Platelet to lymphocyte ratio as a novel prognostic tool for gallbladder carcinoma. World journal of gastroenterology. 2015;21:6675-83. 
14. Bouillanne O, Morineau G, Dupont C, Coulombel I, Vincent JP, Nicolis I, et al. Geriatric Nutritional Risk Index: a new index for evaluating at-risk elderly medical patients. Am J Clin Nutr. 2005;82:777-83.

15. Kanthan R, Senger JL, Ahmed S, Kanthan SC. Gallbladder Cancer in the 21st Century. Journal of oncology. 2015;2015:967472.

16. Zhu AX, Hong TS, Hezel AF, Kooby DA. Current management of gallbladder carcinoma. Oncologist. 2010;15:168-81.

17. Roxburgh CS, McMillan DC. Role of systemic inflammatory response in predicting survival in patients with primary operable cancer. Future oncology. 2010;6:149-63.

18. Gupta D, Lis CG. Pretreatment serum albumin as a predictor of cancer survival: a systematic review of the epidemiological literature. Nutrition journal. 2010;9:69.

19. Body Size Indicators and Risk of Gallbladder Cancer: Pooled Analysis of Individual-Level Data from 19 Prospective Cohort Studies

Campbell PT, Newton CC, Kitahara CM, Patel AV, Hartge P, Koshiol J, et al. Body Size Indicators and Risk of Gallbladder Cancer: Pooled Analysis of Individual-Level Data from 19 Prospective Cohort Studies. Cancer epidemiology, biomarkers \& prevention: a publication of the American Association for Cancer Research, cosponsored by the American Society of Preventive Oncology. 2017;26:597606.

20. Kang J, Lee SH, Son JH, Lee JW, Choi YH, Choi JH, et al. Body mass index and weight change during initial period of chemotherapy affect survival outcome in advanced biliary tract cancer patients. PloS one. 2018;13:e0195118.

21. Cavallin F, Scarpa M, Cagol M, Alfieri R, Castoro C. Low perioperative serum prealbumin predicts early recurrence after curative pulmonary resection for non-small-cell lung cancer. World journal of surgery. 2013;37:2005.

22. Anandavadivelan $P$, Lagergren $P$. Cachexia in patients with oesophageal cancer. Nature reviews Clinical oncology. 2016;13:185-98.

\section{Tables}

Table 1. The comparison of the clinical and pathologic between survivors and non-survivor groups. 


\begin{tabular}{|c|c|c|c|c|}
\hline & overall & Survivors & Non-survivors & $P$ value \\
\hline Case n $(\%)$ & 202 & 108 & 94 & \\
\hline Age, years & $68.54 \pm 11.02$ & $66.81 \pm 10.87$ & $70.52 \pm 13.91$ & 0.017 \\
\hline Female (\%) & $68.3 \%$ & $70.4 \%$ & $66.0 \%$ & 0.546 \\
\hline Height, cm & $159.56 \pm 7.44$ & $159.39 \pm 7.69$ & $159.76 \pm 7.16$ & 0.728 \\
\hline Weight, kg & $58.31 \pm 9.87$ & $56.42 \pm 8.96$ & $59.96 \pm 10.36$ & 0.011 \\
\hline BMI, $\mathrm{kg} / \mathrm{m}^{2}$ & $22.87 \pm 3.29$ & $23.58 \pm 3.53$ & $22.05 \pm 2.80$ & 0.001 \\
\hline GNRI & $100.08 \pm 10.69$ & $104.12 \pm 9.80$ & $95.44 \pm 9.78$ & $<0.001$ \\
\hline Hemoglobin mean, $\mathrm{g} / \mathrm{L}$ & $124.94 \pm 17.97$ & $127.12 \pm 14.43$ & $122.43 \pm 21.13$ & 0.044 \\
\hline Serum albumin mean, $g / L$ & $38.30 \pm 5.18$ & $40.10 \pm 4.19$ & $36.23 \pm 5.44$ & $<0.001$ \\
\hline NLR & & & & 0.112 \\
\hline$<2.6$ & $61.4 \%$ & $66.7 \%$ & $55.3 \%$ & \\
\hline$\geq 2.6$ & $38.6 \%$ & $33.3 \%$ & $44.7 \%$ & \\
\hline Serum CA19-9 $(\mathrm{U} / \mathrm{mL})>37$ & & & & $<0.001$ \\
\hline No & $58.9 \%$ & $72.2 \%$ & $43.6 \%$ & \\
\hline Yes & $41.1 \%$ & $27.8 \%$ & $56.4 \%$ & \\
\hline Serum CEA level $(\mathrm{ng} / \mathrm{mL})>5$ & & & & 0.006 \\
\hline No & $77.5 \%$ & $86.0 \%$ & $67.5 \%$ & \\
\hline Yes & $22.5 \%$ & $14.0 \%$ & $32.5 \%$ & \\
\hline $\mathrm{T}(\%)$ & & & & $<0.001$ \\
\hline I-II & $34.9 \%$ & $48.0 \%$ & $20.9 \%$ & \\
\hline III-IV & $65.1 \%$ & $52.0 \%$ & $79.1 \%$ & \\
\hline Gallstones (\%) & & & & 0.077 \\
\hline No & $39.9 \%$ & $46.1 \%$ & $33.0 \%$ & \\
\hline Yes & $60.1 \%$ & $53.9 \%$ & $67.0 \%$ & \\
\hline Tumor size $(\mathrm{cm})>3(\%)$ & & & & 1.000 \\
\hline No & $53.2 \%$ & $53.6 \%$ & $52.9 \%$ & \\
\hline Yes & $46.8 \%$ & $46.4 \%$ & $47.1 \%$ & \\
\hline Differentiation of GBC (\%) & & & & $<0.001$ \\
\hline Poor/unknown & $37.4 \%$ & $24.2 \%$ & $52.5 \%$ & \\
\hline
\end{tabular}




\begin{tabular}{lcccc} 
Well/moderate & $62.6 \%$ & $75.8 \%$ & $47.5 \%$ & \\
TNM stage (\%) & & & & \\
I-II & $30.5 \%$ & $46.2 \%$ & $12.8 \%$ & \\
III-IV & $69 ; .5$ & $53.8 \%$ & $87.2 \%$ & 0.046 \\
Lymph node metastases (\%) & & & & \\
No & $75.3 \%$ & $80.8 \%$ & $69.2 \%$ & $<0.001$ \\
Yes & $24.7 \%$ & $19.2 \%$ & $30.8 \%$ & \\
GNRI & & & & \\
$\geq 100$ & $49.0 \%$ & $68.5 \%$ & $26.6 \%$ & \\
$<100$ & $51.0 \%$ & $31.5 \%$ & $73.4 \%$ & \\
\hline
\end{tabular}

Table 2 Clinical and pathologic characteristics of the 202 study patients. 


\begin{tabular}{|c|c|c|c|c|}
\hline & overall & High GNRI ( $\geq 100)$ & Low GNRI $(<100)$ & $\mathrm{P}$ value \\
\hline Case n (\%) & 202 & $99(49.0 \%)$ & $103(51.0 \%)$ & \\
\hline Age, years & $68.54 \pm 11.02$ & $65.83 \pm 10.08$ & $71.15 \pm 11.30$ & 0.001 \\
\hline Female (\%) & $68.3 \%$ & $77.8 \% \%$ & $59.2 \%$ & 0.006 \\
\hline BMI, $\mathrm{kg} / \mathrm{m}^{2}$ & $22.87 \pm 3.29$ & $24.49 \pm 3.12$ & $21.30 \pm 2.65$ & $<0.001$ \\
\hline Hemoglobin mean, $g / L$ & $124.94 \pm 17.97$ & $130.02 \pm 12.45$ & $120.05 \pm 20.03$ & $<0.001$ \\
\hline Serum albumin mean, $g / L$ & $38.30 \pm 5.18$ & $42.02 \pm 2.82$ & $34.73 \pm 4.34$ & $<0.001$ \\
\hline NLR & & & & $<0.001$ \\
\hline$<2.6$ & $61.4 \%$ & $78.8 \%$ & $44.7 \%$ & \\
\hline$\geq 2.6$ & $38.6 \%$ & $21.2 \%$ & $55.3 \%$ & \\
\hline Serum CA19-9 $(\mathrm{U} / \mathrm{mL})>37$ & & & & 0.028 \\
\hline No & $58.9 \%$ & $67.9 \%$ & $50.9 \%$ & \\
\hline Yes & $41.1 \%$ & $32.1 \%$ & $49.4 \%$ & \\
\hline Serum CEA level $(\mathrm{ng} / \mathrm{mL})>5$ & & & & 0.467 \\
\hline No & $77.5 \%$ & $80.2 \%$ & $74.7 \%$ & \\
\hline Yes & $22.5 \%$ & $19.8 \%$ & $25.3 \%$ & \\
\hline $\mathrm{T}(\%)$ & & & & 0.286 \\
\hline I-II & $34.9 \%$ & $39.1 \%$ & $30.9 \%$ & \\
\hline III-IV & $65.1 \%$ & $60.9 \%$ & $69.1 \%$ & \\
\hline Gallstones (\%) & & & & 0.048 \\
\hline No & $39.9 \%$ & $46.7 \%$ & $33.7 \%$ & \\
\hline Yes & $60.1 \%$ & $53.3 \%$ & $66.3 \%$ & \\
\hline Tumor size $(\mathrm{cm})>3(\%)$ & & & & 1.000 \\
\hline No & $53.2 \%$ & $53.8 \%$ & $52.7 \%$ & \\
\hline Yes & $46.8 \%$ & $46.2 \%$ & $47.3 \%$ & \\
\hline Differentiation of GBC (\%) & & & & 0.046 \\
\hline Poor/unknown & $37.4 \%$ & $31.8 \%$ & $47.0 \%$ & \\
\hline Well/moderate & $62.6 \%$ & $68.2 \%$ & $53.0 \%$ & \\
\hline TNM stage (\%) & & & & 0.357 \\
\hline I-II & $30.5 \%$ & $34.0 \%$ & $27.2 \%$ & \\
\hline
\end{tabular}



III-IV
69;.5
$66.0 \%$
$72, .8 \%$

Lymph node metastases (\%)

0.615

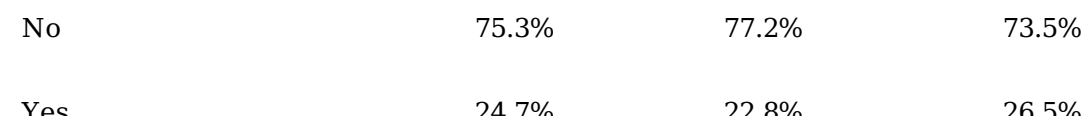

$24.7 \%$

$22.8 \%$

$26.5 \%$

Table 3 Univariate and multivariate analyses of prognostic factors with overall survival.

\begin{tabular}{|c|c|c|c|c|}
\hline \multirow[t]{2}{*}{ Variables } & \multicolumn{2}{|c|}{ Univariate analysis } & \multicolumn{2}{|c|}{ Multivariate analysis } \\
\hline & HR $(95 \% \mathrm{CI})$ & P-value & HR (95\% CI) & P-value \\
\hline Age & 1.026 (1.006-1.046) & 0.009 & $1.030(1.001-1.061)$ & 0.045 \\
\hline Sex male & $1.195(0.779-1.832)$ & 0.414 & & \\
\hline serum CA 19-9(U/mL)>37 & $2.304(1.470-3.612)$ & $<0.001$ & & \\
\hline Serum CEA $(\mathrm{ng} / \mathrm{mL})>5$ & $1.904(1.192-3.041)$ & 0.007 & & \\
\hline Hemoglobin & $1.192(1.098-1.214)$ & 0.038 & 1.025 (1.001-1.049) & 0.043 \\
\hline NLR $>2.6$ & $1.447(0.963-2.174)$ & 0.075 & & \\
\hline Gallstones & $1.271(0.821-1.970)$ & 0.282 & & \\
\hline Tumor size $>3 \mathrm{~cm}$ & $1.021(0.639-1.633)$ & 0.930 & & \\
\hline T III-IV & $1.630(1.253-2.121)$ & $<0.001$ & & \\
\hline Poor differentiation & $2.323(1.496-3.607)$ & $<0.001$ & $2.073(1.079-4.391)$ & 0.047 \\
\hline Lymph node metastases & $1.567(1.004-2.448)$ & 0.048 & & \\
\hline TNM stage III+IV & 3.688 (2.011-6.764) & $<0.001$ & $8.182(1.727-38.776)$ & 0.008 \\
\hline GNRI $<100$ & $3.656(2.308-5.790)$ & $<0.001$ & $2.386(1.170-4.868)$ & 0.017 \\
\hline
\end{tabular}

Table4 Univariate and multivariate analyses of prognostic factors associated with RFS. 


\begin{tabular}{|c|c|c|c|c|}
\hline \multirow[t]{2}{*}{ Variables } & \multicolumn{2}{|c|}{ Univariate analysis } & \multicolumn{2}{|c|}{ Multivariate analysis } \\
\hline & HR (95\%CI) & P-value & HR (95\% CI) & $\mathrm{P}$-value \\
\hline Age & $1.016(0.998-1.034)$ & 0.074 & & \\
\hline Sex male & $0.808(0.539-1.210)$ & 0.301 & & \\
\hline BMI & $0.898(0.842-0.959)$ & 0.001 & & \\
\hline serum CA $19-9(\mathrm{U} / \mathrm{mL})>37$ & $1.928(1.263-2.945)$ & 0.002 & & \\
\hline Serum CEA $(\mathrm{ng} / \mathrm{mL})>5$ & $1.631(1.025-2.593)$ & 0.039 & & \\
\hline Hemoglobin & $0.994(0.982-1.006)$ & 0.306 & & \\
\hline NLR $>2.6$ & $1.527(1.036-2.251)$ & 0.032 & & \\
\hline Gallstones & $1.506(0.995-2.279)$ & 0.053 & & \\
\hline Tumor size $>3 \mathrm{~cm}$ & $0.892(0.570-1.396)$ & 0.617 & & \\
\hline T III-IV & $1.112(0.904-1.368)$ & 0.314 & & \\
\hline Poor differentiation & $1.640(1.084-2.484)$ & 0.019 & & \\
\hline Lymph node metastases & $3.318(1.128-7.048)$ & 0.014 & & \\
\hline TNM stage III+IV & $1.617(1.037-2.520)$ & 0.034 & $3.236(1.051-11.117)$ & 0.049 \\
\hline GNRI $<100$ & 5.395 (3.421-8.508) & $<0.001$ & 7.610 (3.181-18.207) & $<0.001$ \\
\hline
\end{tabular}

\section{Figures}




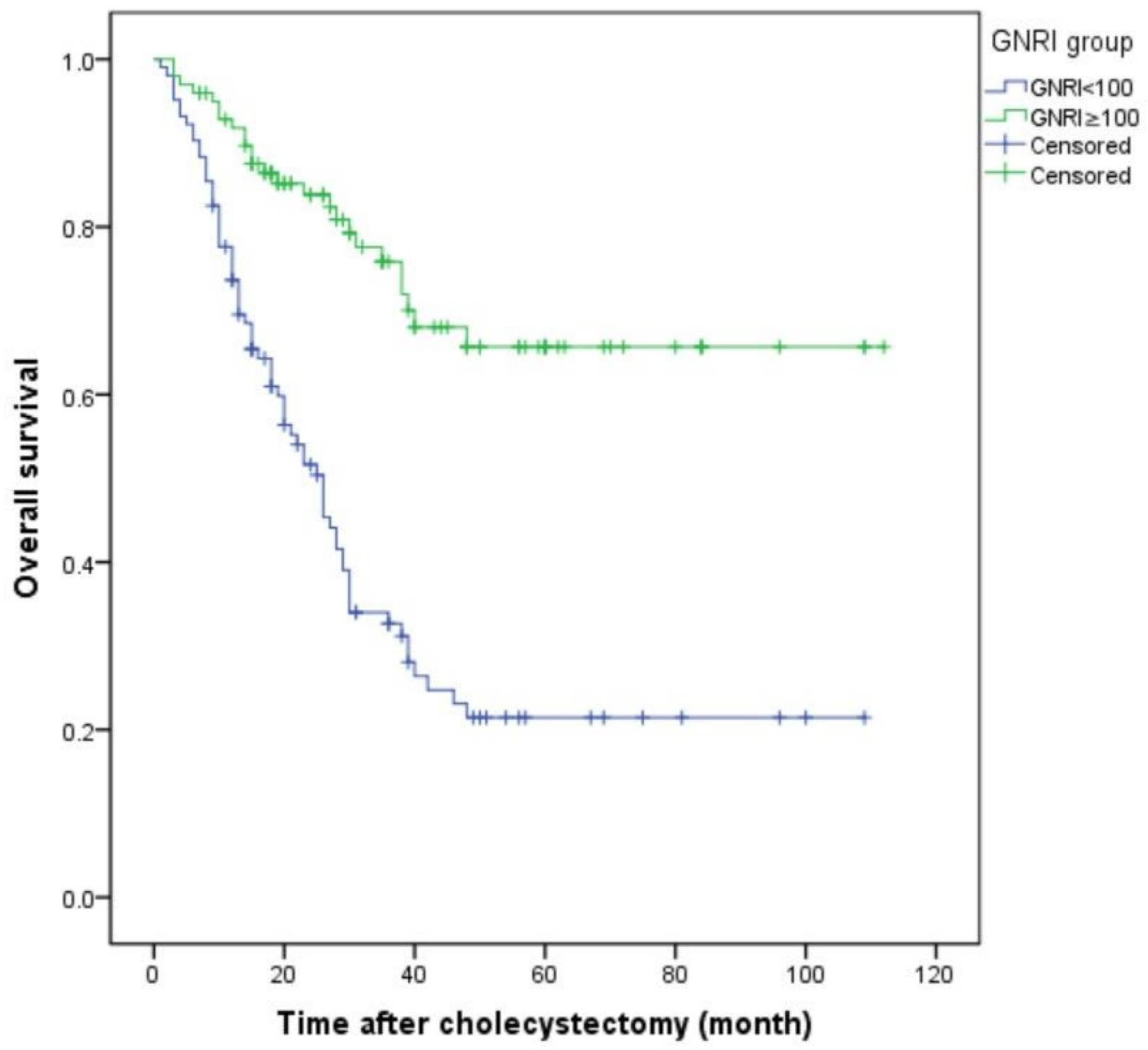

Figure 1

The overall survival rate of GBC patients after GBC radical surgery. Notes: The Kaplan-Meier curve showed significant differences in the probability of total survival after GBC radical surgery in patients with $\mathrm{GNRI}<100$ and $\mathrm{GNRI} \geq 100$. $\mathrm{P}<0.001$ (log-rank test). 


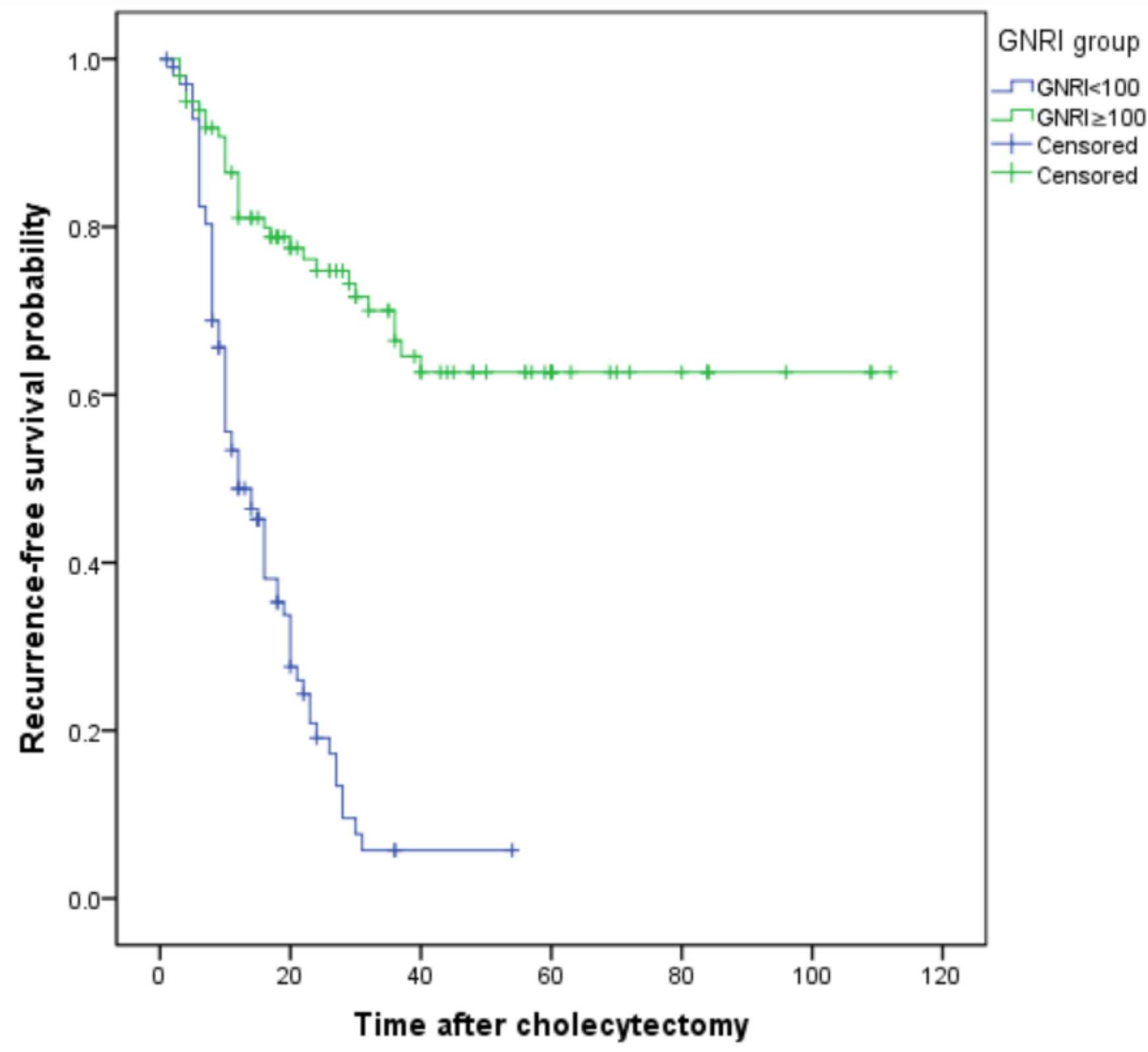

Figure 2

RFS of patients with GBC after GBC radical surgery. Notes: Kaplan-Meier curves show significant difference in RFS probability after GBC radical surgery in patients with preoperative high GNRI and low GNRI levels. $P<0.001$ (log-rank test). 
(a)

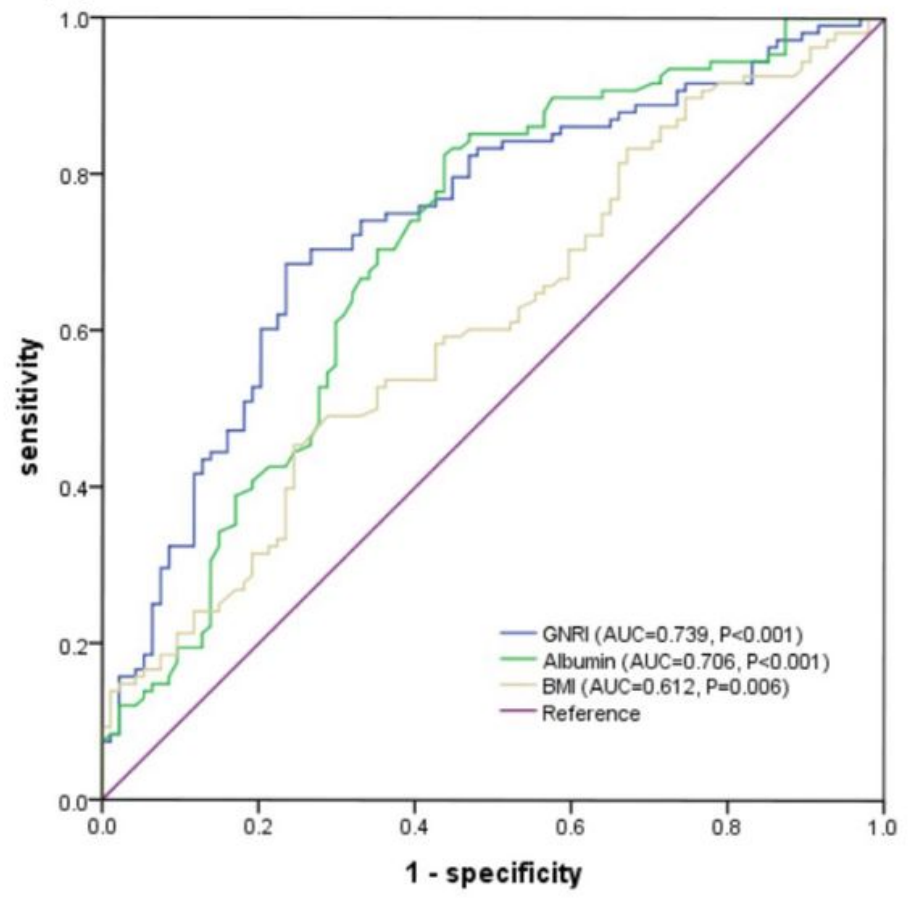

(b)

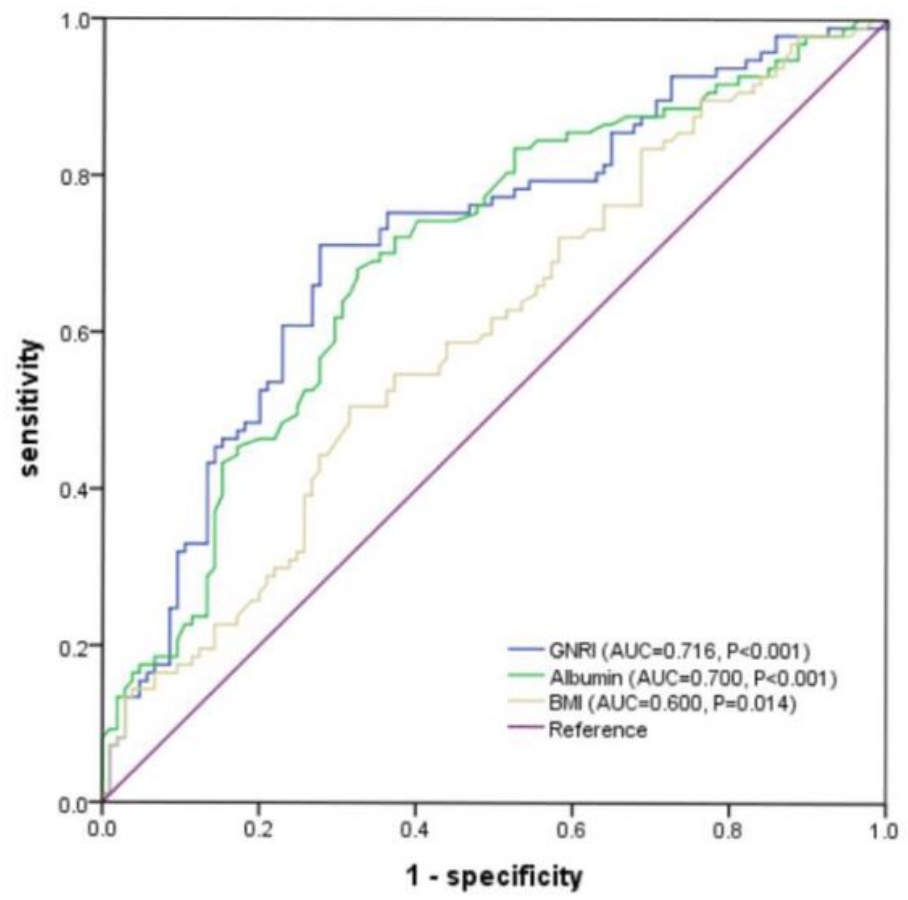

Figure 3

(a) Receiver operating characteristic curve analysis based on GNRI, albumin and BMI for overall survival (b) Receiver operating characteristic curve analysis based on GNRI, albumin and BMI for RFS. 